ARTICLE

DOI: $10.1038 / s 41467-018-05829-7$

\title{
Overexpressing IncRNA LAIR increases grain yield and regulates neighbouring gene cluster expression in rice
}

Ying Wang ${ }^{1,2}$, Xiaojin Luo ${ }^{1}$, Fan Sun ${ }^{1}$, Jianhua $\mathrm{Hu}^{2,3}$, Xiaojun $\mathrm{Zha}^{4}$, Wei Su${ }^{1,5}$ \& Jinshui Yang ${ }^{1}$

Long noncoding RNAs (IncRNAs) are essential regulators of gene expression in eukaryotes. Despite increasing knowledge on the function of IncRNAs, little is known about their effects on crop yield. Here, we identify a IncRNA transcribed from the antisense strand of neighbouring gene LRK (leucine-rich repeat receptor kinase) cluster named LAIR (LRK Antisense Intergenic RNA). LAIR overexpression increases rice grain yield and upregulates the expression of several $L R K$ genes. Additionally, chromatin immunoprecipitation assay results indicate $\mathrm{H} 3 \mathrm{~K} 4 \mathrm{me} 3$ and $\mathrm{H} 4 \mathrm{~K} 16 \mathrm{ac}$ are significantly enriched the activated LRK1 genomic region. LAIR binds histone modification proteins OsMOF and OsWDR5 in rice cells, which are enriched in LRK1 gene region. Moreover, LAIR is demonstrated to bind $5^{\prime}$ and $3^{\prime}$ untranslated regions of $L R K 1$ gene. Overall, this study reveals the role of IncRNA LAIR in regulating rice grain yield and IncRNAs may be useful targets for crop breeding.

\footnotetext{
${ }^{1}$ State Key Laboratory of Genetic Engineering, Institute of Genetics, School of Life Sciences, Fudan University, Shanghai 200438, China. ${ }^{2}$ Research Center for Ecological Science and Technology, Fudan Zhangjiang Institute, Shanghai 201203, China. ${ }^{3}$ State Key Laboratory of Molecular Engineering of Polymers, Department of Macromolecular Science, Fudan University, Shanghai 200433, China. ${ }^{4}$ College of Chemistry and Life Science, Zhejiang Normal University, Jinhua 321004, China. ${ }^{5}$ State Key Laboratory of Genetic Engineering, Institute of Plant Biology, Department of Biochemistry, School of Life Sciences, Fudan University, Shanghai 200438, China. Correspondence and requests for materials should be addressed to W.S. (email: weisu@fudan.edu.cn)

or to J.Y. (email: jsyang@fudan.edu.cn)
} 
$\mathrm{R}$ ice (Oryza sativa L.) is a staple food crop for more than 3 billion people worldwide ${ }^{1}$. It is also considered as one of the most important model monocotyledonous plants for genome research due to its small genome size and considerable synteny with other cereal genomes ${ }^{2,3}$. Yield is a crucial agronomic trait for cereal crops' genetic improvement. Its improvement relies on the manipulation of a range of component traits such as number of panicles, grains per plant, and 1000-grain weight, which often exhibit continuous phenotypic variations. It has been shown that these traits are regulated by quantitative trait loci (QTLs), whose effects are usually mediated by at least two genes and environmental conditions.

The traditional definition of a gene has recently been challenged by the discovery of noncoding RNAs (ncRNAs), which include functional transcripts encoded by a specific genome region. A large-scale sequencing analysis reveals that most of the eukaryotic genome is transcribed ${ }^{4}$, and large repertoires of RNAs are generated, including short (22-33 nucleotides) and long ( $>200$ nucleotides) ncRNAs. Long noncoding RNAs (lncRNAs), which lack any obvious open reading frames $(\mathrm{ORFs})^{5-7}$, function as key regulators of diverse mechanisms in a range of biological processes $^{6,8,9}$. They may regulate the expression of neighbouring genes with cis-acting (close to the transcription site) or transacting (multiple loci throughout the genome) mechanisms ${ }^{10}$. Approximately one-third of lncRNAs can interact with multiple protein partners ${ }^{11}$. The concept of lncRNAs serving as molecular scaffolds that help assemble and target the chromatin-modifying complex to specific genomic loci for the regulation of gene expression is likely part of a globally emerging mechanistic theme ${ }^{6,12}$. One major theme involves the regulatory role of lncRNAs regarding the expression of nearby protein-coding genes or gene clusters. For example, HOX transcript antisense RNA (HOTAIR), one of the first identified trans-acting lncRNAs, binds to multiple genomic loci of HOXD genes and serves as a molecular scaffold that enables the recruitment of chromatinmodifying complexes to repress the expression of HOXD genes $^{13,14}$. In Drosophila melanogaster, the dosage compensation complex with lncRNAs [RNA on X1 (roX1) and RNA on X2 (roX2)] that combine with proteins [male-specific lethal-1 (MSL1), MSL-2, MSL-3, Males-absent-on-the-first (MOF), and Maleless (MLE)] acetylates H4K16 so that chromatin can assume a more open conformation in the hyperactive cells of male fruit flies ${ }^{15-17}$. Genomic occupancy maps of roX RNAs have also been done to show the targeting of $\mathrm{X}$ chromosomes ${ }^{18}$.

In plants, it is known that lncRNAs can regulate gene silencing, flowering time, reproduction, stress responses, organogenesis in roots, and photomorphogenesis in seedlings ${ }^{19-24}$. Because the overwhelming majority of well-characterized plant lncRNAs with established functions were studied in Arabidopsis thaliana, our understanding of the mechanisms regulating lncRNAs in crop species remains limited. Thus, elucidating the mechanisms of action for the rice lncRNAs may clarify the functions of ncRNAs in different species and provide useful information for the breeding of food crops. We previously detected and cloned a leucine-rich repeat receptor kinase $(L R K)$ gene cluster from a QTL associated with increased grain yield in rice ${ }^{25}$. In this study, we identify a lncRNA transcript (LRK Antisense Intergenic RNA, $L A I R)$ from the antisense strand of the $L R K$ gene cluster. Transgenic lines, in which LAIR is silenced, exhibit inhibited growth and low expression levels for all $L R K$ genes. In contrast, considerable increases in total grain yield and upregulated expression of some members of the $L R K$ gene cluster are observed in lines overexpressing LAIR. Moreover, luciferase reporter assays reveal that $L A I R$ is capable of differentially activating promoters of the LRKs gene. Meanwhile, an analysis of chromatin modifications related to $L R K$ genes indicates $\mathrm{H} 3 \mathrm{~K} 4 \mathrm{me} 3$ and $\mathrm{H} 4 \mathrm{~K} 16 \mathrm{ac}$ are enriched in transcriptionally active genes. Next, LAIR binds histone modification proteins OsMOF and OsWDR5 (WD repeat domain 5) in rice cells, which are detected enrichment in the LRK1 gene region. Additionally, ChIRP assays reveal that LAIR binds $5^{\prime}$ and $3^{\prime}$ UTR genomic regions of the $L R K 1$ gene. These results imply that LAIR overexpression increases rice yield and regulates the expression of neighbouring $L R K$ gene cluster.

\section{Results}

Identification of the antisense IncRNA LAIR. We previously identified a QTL for improved rice yield consisting of several tandemly arranged intronless $L R K$ gene copies in a $51.3-\mathrm{kb}$ region of chromosome $2^{25}$. The $L R K$ gene cluster in the $O$. sativa ssp. indica MH63 genome was subsequently sequenced. Genomic and transcriptomic analyses revealed the $L R K$ gene cluster in MH63 contained eight highly similar $L R K$ genes (LRK1-8), which were transcribed normally, with the exception of LRK5 (Fig. 1a).

An antisense transcript encoded by the $5^{\prime}$ terminal region of the $L R K$ gene cluster was named LAIR. The LRK1 gene was located in the first intron of $L A I R$, and there was no overlap between the two antisense transcripts (Fig. 1a). The $5^{\prime}$ and $3^{\prime}$ ends of $L A I R$ were determined by rapid amplification of cDNA ends (RACE) (Supplementary Fig. 3). We observed that LAIR contains a $5^{\prime}$ cap structure and a polyadenylated $3^{\prime}$ end, which is typical of mRNAs. Thus, it is likely that RNA polymerase II (RNAPII) is responsible for the transcription of LAIR. The spatial and temporal expression of LAIR was analyzed, LAIR showed relatively high levels in 3-leaf-stage shoot, flowering-stage node, pistil and caryopsis (Supplementary Fig. 1). Additionally, up to 10 alternative spliced isoforms of LAIR were identified and sequenced (JX512719-JX512728). All splicing events were detected in the second exon of $L A I R$, and consisted of the shuffling of specific motifs rather than random events. The most enriched LAIR RNA (JX512726) was $1797 \mathrm{bp}$ long, while the shortest LAIR RNA (JX512719) was 1585 bp long. The corresponding genomic and transcript sequences were up to $7.3 \mathrm{~kb}$ and approximately $1.8 \mathrm{~kb}$ long, respectively (Supplementary Fig. 2 and Fig. 1a).

We evaluated the coding potential of LAIR. The ORF Finder online tool (https://www.ncbi.nlm.nih.gov/orffinder/) was used to predict ORFs based on LAIR isoform JX512726. As indicated in Fig. 2a, the termination codon was distributed in all three frames, and only a few sequences encoding short peptides were detected. The two longest peptides comprised 133 and 104 amino acids. Furthermore, there were no functional domain matches for any of the peptides according to homology searches of large databases of protein families and domains ( Pfam $^{26}$ and SMART $\left.{ }^{27}\right)$. The possibility that these peptides function as small proteins was assessed by the following genetic investigations. An analysis of the protein-coding potential of transcripts ${ }^{28}$ indicated that all 10 alternative spliced isoforms of LAIR represented ncRNAs (Fig. 2b). Additionally, the DNA sequences of the different alternatively spliced $L A I R$ isoforms had diverse coding frames. Therefore, we hypothesized that there is no functional constraint associated with the LAIR coding regions.

To summarize, we identified an antisense lncRNA (LAIR) from the $5^{\prime}$ terminal region of the $L R K$ gene cluster; further analyses indicated its association with rice grain yield.

LAIR affects rice yield traits. To test whether LAIR is functional in individual plants, we conducted genetic transformation experiments. Full-length cDNA sequences of sense or antisense LAIR (JX512726) were cloned and transformed into rice MH63 under the control of the 35S Cauliflower Mosaic Virus (CaMV) promoter (35S::LAIR and 35S::anti-LAIR). Compared with the 

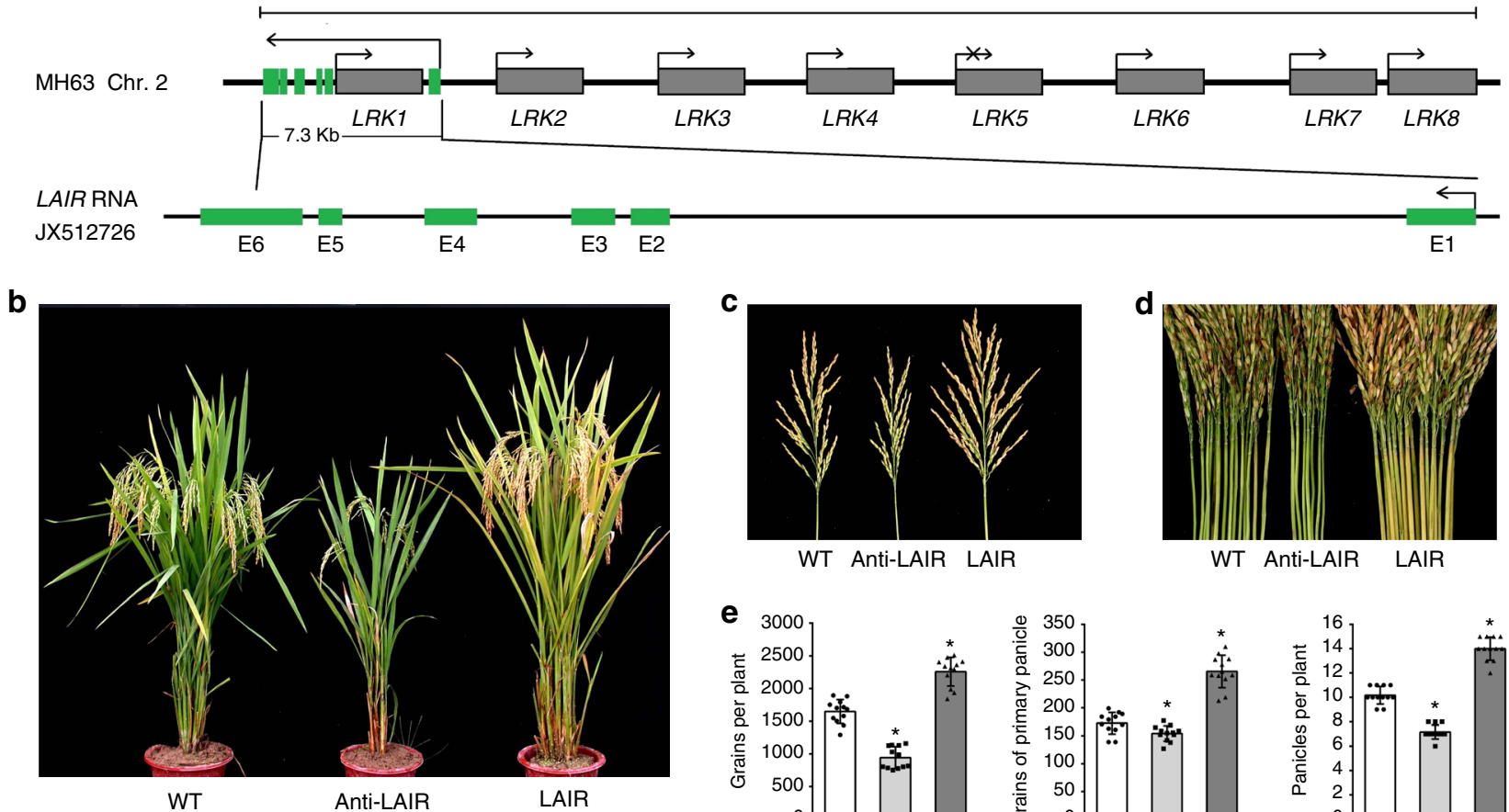

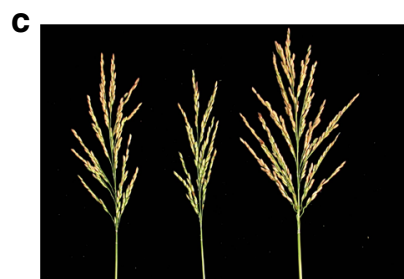

WT Anti-LAIR LAIR

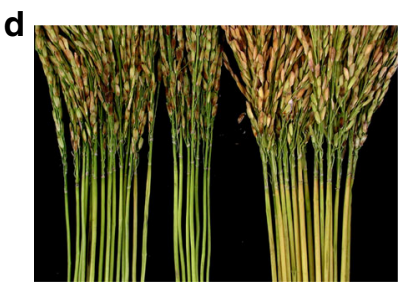

WT Anti-LAIR LAIR
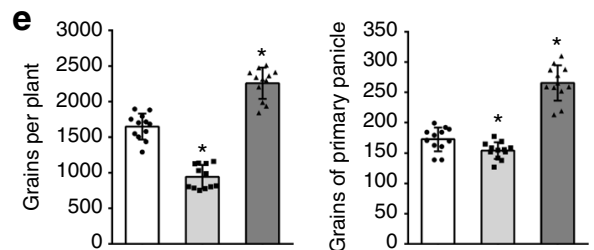

$\square$ WT

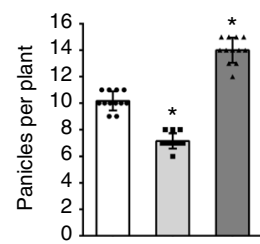

$\square$ Anti-LAIR $\square$ LAIR

Fig. 1 Cloning and characterization of LAIR. a Genome map of the $L R K$ gene cluster and $L A I R$. The $L R K$ genes are located in a 51.3-kb region on chromosome 2 of Nipponbare PAC clone AP007224. LncRNA LAIR (JX512726), which includes six exons, is transcribed from the antisense strand of LRK1. Additionally, LRK1 is in the first LAIR intron, with no overlap between the two transcripts. b-d Phenotypes of wild-type (WT), antisense RNAi-LAIR (Anti-LAIR), and LAIRoverexpressing (LAIR) transgenic rice plants. Growth traits of $T_{3}$ transgenic lines were investigated, including mature plant phenotype (b), primary panicle size (c), and number of panicles per plant (d). e Growth traits of Anti-LAIR and LAIR transgenic lines. Student's $t$-test: ${ }^{\star} P<0.05$. Data are presented as the mean \pm standard deviation $(n=12)$

wild-type control plants, the 35S::LAIR plants had larger primary panicles and more panicles per plant, leading to a considerable increase in total grain yield per plant (Fig. 1b-e). In contrast, the 35S::anti-LAIR transgenic plants exhibited inhibited growth and decreased grain yield. Similar rice growth traits were reported for LRK1-overexpressing transgenic lines ${ }^{29}$. These results demonstrate that LAIR expression affects rice plant growth and grain yield.

The LAIR transcript is long (approximately $1.8 \mathrm{~kb}$ ) and was predicted to lack coding potential according to the ORF and functional domain analyses. To investigate whether LAIR functions as a lncRNA, we mutated the sequences encoding the two longest peptides (133 and 104 amino acids according to the JX512726 sequence). Two LAIR mutant vectors were constructed, with a single nucleotide mutation introducing a premature stop codon in the LAIR-MU1 mutant, and a single deleted nucleotide after the start codon (ATG) in the LAIR-MU2 mutant (Fig. 2c). We analyzed transgenic rice MH63 plants carrying the 35S:: LAIR-MU1 and 35S::LAIR-MU2 constructs and observed that the morphological characteristics and grain yield of these mutants LAIR lines were similar to those of the wild-type LAIRoverexpressing (35S::LAIR) transgenic lines (Fig. $2 \mathrm{~d}-\mathrm{f}$ ). These results implied that LAIR functions were not dependent on the encoded products. Thus, LAIR likely serves as a lncRNA that regulates rice plant growth and increases grain yield.

LAIR influences the transcription of $L R K$ gene cluster. An earlier study that confirmed non-coding loci can affect the expression of neighbouring protein-coding genes was critical for the functional characterization of ncRNAs ${ }^{30}$. Additionally, the 35S::LAIR transgenic rice lines exhibited improved growth traits that were consistent with the growth characteristics of the LRK1overexpressing transgenic rice lines. Thus, a qRT-PCR analysis was used to investigate the expression of LRK genes in LAIRassociated transgenic rice lines. In the LAIR-overexpressing $35 \mathrm{~S}::$ LAIR lines, the $L R K$ genes were differentially expressed. The LRK1 and LRK4 transcript levels were high, while the expression levels for the other $L R K$ genes remained unchanged or decreased slightly (Fig. 3a). Furthermore, LRK genes expression in the LAIR mutant lines with the 35S::LAIR-MU1 or 35S::LAIR-MU2 constructs was similar to that in the 35S::LAIR lines (Fig. 3b). Accordingly, LAIR may have a key role influencing the transcriptional regulation of $L R K$ genes. All $L R K$ genes were expressed at relatively low levels in the 35S::anti-LAIR lines, suggesting that LAIR was crucial for the expression of the $L R K$ gene cluster (Fig. 3d).

To clarify the regulatory effects of LAIR on the expression of LRK genes, rice protoplasts were transformed with sense or antisense LAIR expression vectors along with the vector harboring the $L R K$ promoter-driven luciferase reporter gene. The LUC/REN signal ratios suggested that wild-type LAIR (LAIR) could significantly promote the promoter activations of LRK1 and LRK4. Simultaneously, mutant LAIR (LAIR-MU1) showed similar activity ability on LRKs (Fig. 3c). On the contrary, antisense LAIR (anti-LAIR) suppressed the promoter activations of all the LRKs (Fig. 3e). The different regulatory effects between sense LAIR and antisense LAIR may be caused by the fact that sense LAIR transformation only contained one isoform (JX512726) of alternative spliced LAIR, and antisense LAIR could 
interfere all isoforms. Together, our results implied that LAIR was capable of activating the $L R K$ promoter in vivo, with different regulatory effects among different $L R K$ loci.

LAIR overexpression changes histone modification marks. Recent advances revealed the broad functional repertoire of lncRNAs, including their effects on chromatin modifications and epigenetic changes to specific genomic loci that regulate the expression of neighbouring protein-coding genes ${ }^{30}$. To assess whether altered LAIR expression results in changes of histone modifications, we completed a chromatin immunoprecipitation (ChIP) assay to investigate the modification status of $L R K$ loci. We analyzed the histone modifications associated with transcriptional activation, including trimethylated histone $\mathrm{H} 3$ lysine 4 (H3K4me3), acetylated H4K5 (H4K5ac), H3K9ac, H4K16ac, and $\mathrm{H} 3 \mathrm{~K} 27 \mathrm{ac}^{31-34}$. We mainly focused on LRK1 because its transcription was increased in the 35S::LAIR lines, while LRK3 was used as a control because its transcription was unaffected by LAIR expression (Fig. 3a-c).

Our ChIP-qRT-PCR results indicated that H3K4me3 and H4K16ac were unaffected at the LRK3 locus, but were obviously enriched at the promoter, $3^{\prime}$ untranslated region, and LRK1 gene body in the $L A I R$-overexpressing line relative to the levels in the wild-type plants (Fig. 4b). These observations suggested that the overexpression of LAIR increased the H3K4me3 and H4K16ac levels at the $L R K 1$ chromatin region, which was consistent with the upregulated transcription of $L R K 1$ in the 35S::LAIR lines. In contrast, when enrichments of $\mathrm{H} 3 \mathrm{~K} 4 \mathrm{me} 3$ and $\mathrm{H} 4 \mathrm{~K} 16 \mathrm{ac}$ were analyzed in the 35S::anti-LAIR lines, reduction were observed both at the LRK1 and LRK3 locus (Fig. 4c), which were consistent with the suppression of anti-LAIR to $L R K s$ promoter (Fig. $3 \mathrm{~d}, \mathrm{e}$ ). Additionally, analysis of histone modifications on LRK4 which was also activated by LAIR (Fig. $3 \mathrm{a}-\mathrm{c}$ ) was completed. The $\mathrm{H} 3 \mathrm{~K} 4 \mathrm{me} 3$ and H4K16ac levels were increased in the 35S::LAIR lines, and reduced in the 35S::anti-LAIR lines (Supplementary Fig. 5). The results could exclude the possibility that the changes of H3K4me3 and H4K16ac levels at LRK1 locus might be caused by the transcription of $L A I R$ itself. Taken together, the results suggested that $L A I R$ overexpression resulted in altered expression of specific $L R K$ genes and correlated with the changes in histone modifications.

LAIR binds OsMOF and OsWDR5 that target LRK1 genomic region. A considerable proportion of lncRNAs was recently confirmed to associate with chromatin-modifying complexes and modulate gene transcription ${ }^{11}$. Because of the histone modification changes of H3K4me3 and H4K16ac in our ChIP assay (Fig. 4b, c), we tested the interaction between LAIR and chromatin-modifying complex proteins. The MOF enzyme is the primary histone $\mathrm{H} 4 \mathrm{~K} 16$ acetyltransferase in mammalian cells ${ }^{35}$, and is reportedly a component of MSL and the nonspecific lethal (NSL) complex $^{36,37}$. An increased abundance of $\mathrm{H} 3 \mathrm{~K} 4 \mathrm{me} 3$ was also identified in the a
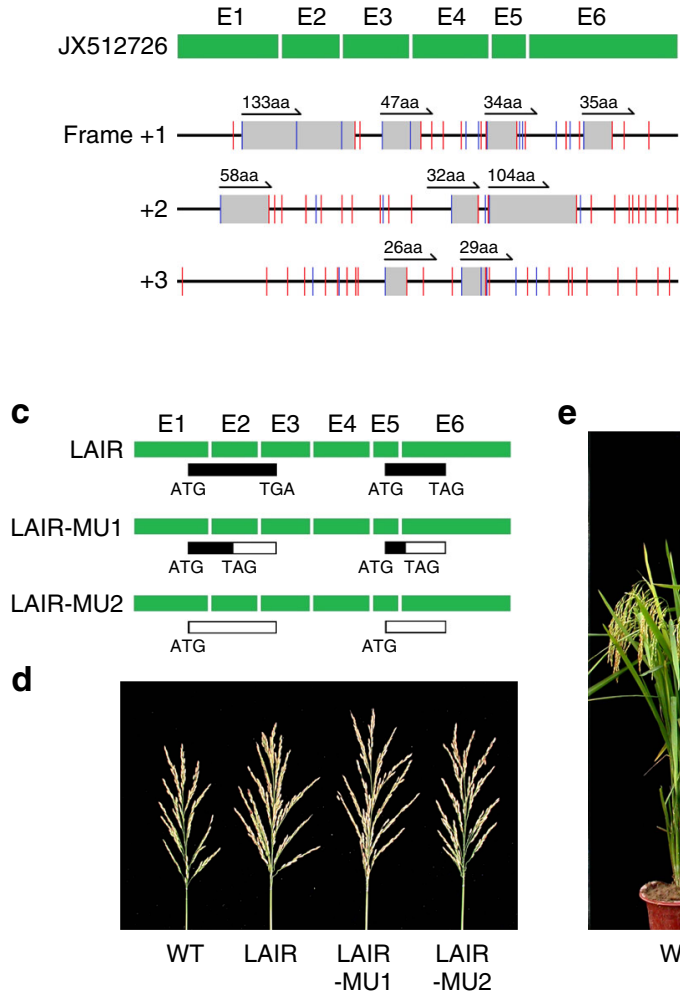

e
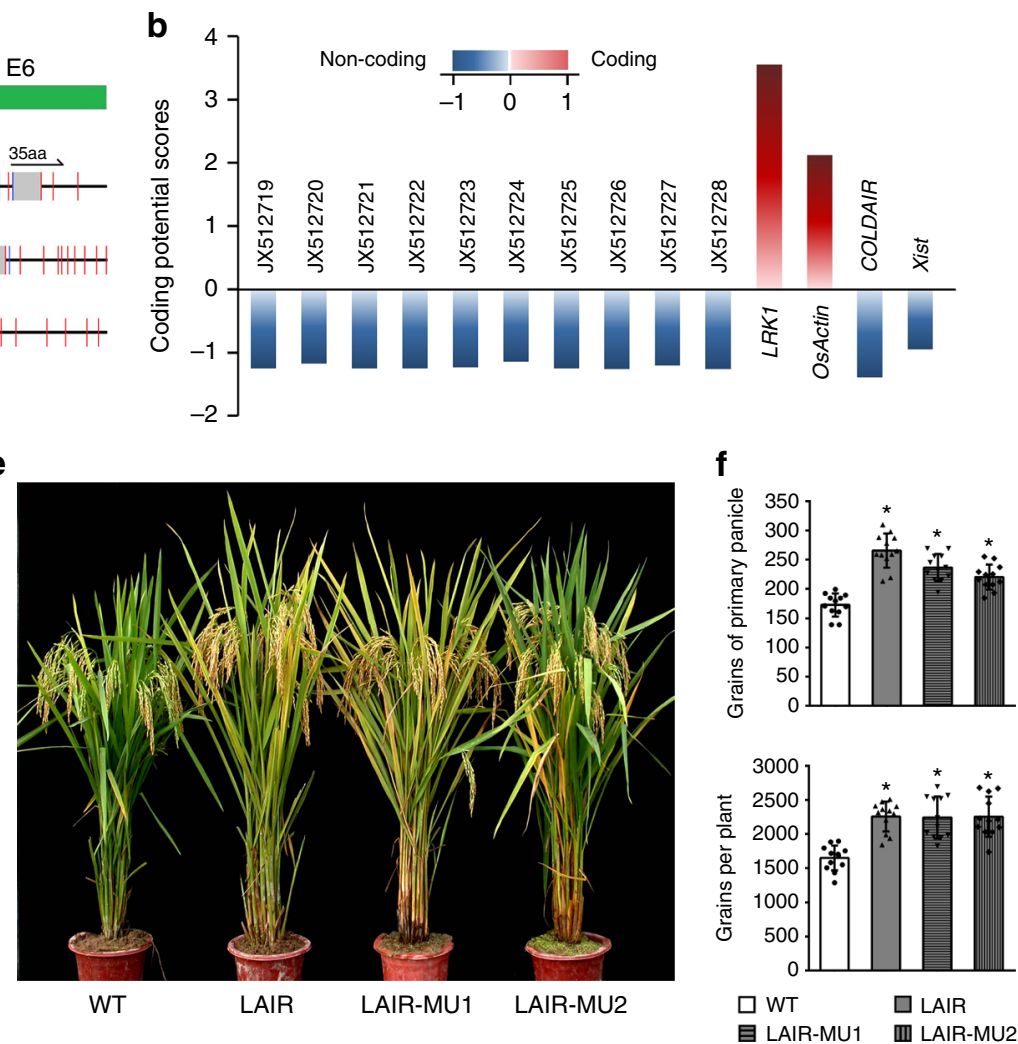

Fig. 2 LAIR coding potential analysis. a Analysis of the LAIR open reading frame (JX512726). All frames (gray boxes) were identified in the three forward frames. The two longest open reading frames encoded 133 and 104 amino acids (aa). Blue and red lines represent the start and stop codons, respectively. $\mathbf{b}$ Analysis of the coding potential of the alternatively spliced LAIR isoforms (Supplementary Fig. 2). Coding potential scores were generated using the CPC program. Transcripts with scores beyond -1 and 1 are marked as non-coding or coding in this CPC classification 28 . LRK1 and OsActin are provided as coding examples, while COLDAIR and Xist represent non-coding examples. c LAIR-mutant vectors were constructed based on the two longest open reading frames, which encoded 133 aa and 104 aa. A single nucleotide mutation introduced a premature stop codon in LAIR-MU1. A single nucleotide was deleted after the start codon (ATG) in LAIR-MU2. d, e Phenotypes of WT, LAIR, and transgenic plants carrying the LAIR mutant vectors. Growth traits of T 3 transgenic lines were investigated, including mature plant phenotype (e) and primary panicle size (d). f Growth traits of LAIR-MU1 and LAIR-MU2 transgenic lines. Student's $t$-test: ${ }^{\star} P<0.05$. Data are presented as the mean \pm standard deviation $(n=12)$ 

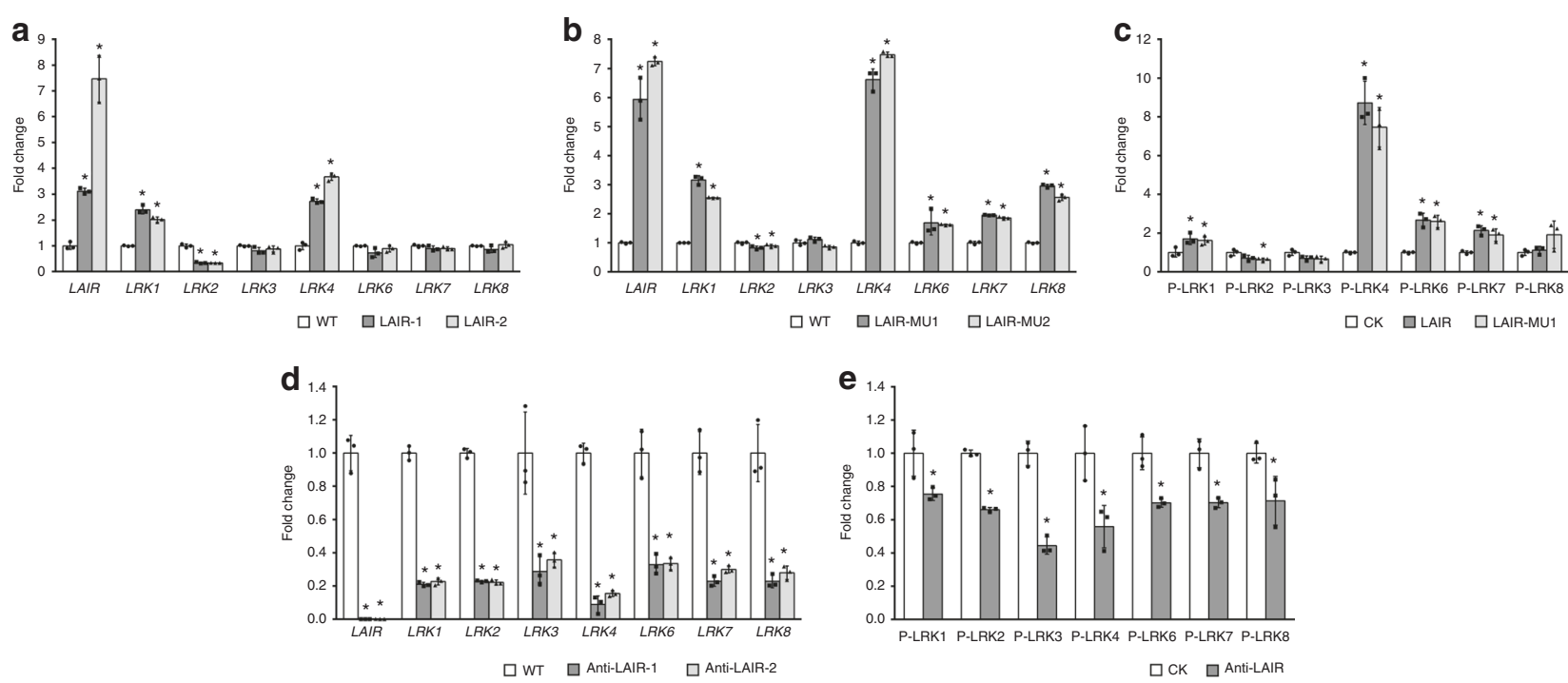

Fig. 3 Differential regulation of $L R K$ gene expression by $L A I R$. $\mathbf{a}, \mathbf{b}, \mathbf{d}$ qRT-PCR analysis of $L R K$ expression in transgenic rice lines. $L A I R$-overexpressing (a, LAIR-1 and LAIR-2), LAIR-mutant (b, LAIR-MU1 and LAIR-MU2), and antisense RNAi-LAIR vectors (d, Anti-LAIR-1 and Anti-LAIR-2). WT: wild-type rice. OsActin was used as a reference control, and gene expression levels in WT lines were used for data normalization. c, e Regulation of the LRK gene promoter by sense or antisense LAIR. Relative LUC activity from the transient expression of the LRK promoter-driven luciferase reporter gene co-infiltrated with sense-LAIR (c, LAIR), LAIR-mutant (c, LAIR-MU1), or antisense LAIR (e, Anti-LAIR) vectors. CK: empty vector control. The CK LUC/REN signal ratios were used for data normalization. All data are presented as the mean \pm standard deviation $(n=3)$. Student's $t$-test: ${ }^{\star} P<0.05$

promoter regions of NSL-targeted genes ${ }^{38}$. Additionally, MLE is a critical protein that associates with ncRNAs to form the MSL complex ${ }^{39,40}$. An NSL component, WDR5, has recently been reported to interact with hundreds of $\operatorname{lncRNAs}{ }^{33,41}$.

Homologs of human MOF (NM_032188), D. melanogaster MLE (NM_057293), and human WDR5 (NM_017588) in rice (OsMOF XM_015789227, OsMLE XM_015756470, and OsWDR5 XM_015772971, respectively) were cloned and fused in-frame with the FLAG tag sequence. Rice protoplasts were co-infiltrated with these genes and the 35S::LAIR vector or 35S::anti-LAIR for a RNA immunoprecipitation (RIP) assay. Our RIP-qRT-PCR results revealed the significant binding of OsMOF and OsWDR5, but not OsMLE, to the $5^{\prime}$ and $3^{\prime}$ regions of LAIR (Fig. $4 \mathrm{~d}$ ). These results were further confirmed by RIP assay using the OsMOFFLAG and OsWDR5-FLAG transgenic rice lines (Supplementary Fig. 4). Thus, LAIR appears to be able to interact with two subunits of the chromatin-modifying complex in rice cells.

Furthermore, to investigate the binding ability of OsMOF and OsWDR5 to LRKs genomic loci, we performed ChIP assay in the OsMOF-FLAG and OsWDR5-FLAG transgenic rice lines. A notable enrichment was detected in the $L R K 1$ region, while the binding levels at the LRK3 locus were similar to those of the controls (Fig. 4e). Our findings confirmed that OsMOF and OsWDR5 can target LRK1 genomic region.

$L A I R$ binds to $L R K$ genomic loci. To investigate the association between genomic DNA region of LRKs and LAIR, we performed chromatin isolation by RNA purification (ChIRP) assay. Successful enrichment of LAIR RNA using biotinylated LAIRantisense DNA (LAIR-asDNA) probes was confirmed by LAIR 5 '-primer qRT-PCR. The result showed that LAIR-asDNA probes retrieved $\sim 65 \%$ of LAIR RNA and undetectable OsActin (Fig. 4f, left panel). Identification of DNA fraction extracted from ChIRP samples showed that LAIR significantly occupied at the $5^{\prime}$ and $3^{\prime}$ untranslated regions of $L R K 1$ compared with $L R K 3$ (Fig. 4f, right panel). These data suggested that LAIR RNA could physically locate to $L R K s$ gene genomic DNA region, with different effects among the $L R K$ loci.

\section{Discussion}

There has recently been considerable interest in the noncoding transcripts in diverse eukaryotes. Accordingly, there has been a rapid increase in the number of well-studied lncRNAs in mammals, while related plant studies have lagged behind. In this study, a lncRNA, LAIR, was identified in rice. Most of the tens of thousands of ncRNA sequences encoded in plant genomes are transcribed by RNAPII, and the majority of the identified plant lncRNAs are polyadenylated ${ }^{42}$. Similarly, LAIR contains a $5^{\prime}$ cap structure and a polyadenylated $3^{\prime}$ end, implying RNAPII is responsible for the transcription of $L A I R$ as well as the nearby protein-coding genes. Moreover, it is likely that most of the uncharacterized lncRNAs help regulate the expression of neighbouring genes ${ }^{43}$. The LAIR sequence is encoded in the antisense strand of the $5^{\prime}$ terminal region of the $L R K$ gene cluster. Additionally, $L R K 1$ is located in the first intron of $L A I R$, with no overlap between the two antisense transcripts (Fig. 1a). Thus, LAIR functions may not involve a direct base-paired interaction with the transcript of the protein-coding $L R K$ genes. Furthermore, we discovered that LAIR overexpression can result in highly upregulated expression of several $L R K$ genes as well as an obvious increase in rice grain yield. Meanwhile, the increased transcription of antisense $L A I R$ downregulates the expression of all $L R K$ genes and inhibits plant growth (Figs. $1 \mathrm{~b}-\mathrm{e}$ and $3 \mathrm{a}, \mathrm{d}$ ). Considering exogenous $L A I R$ was randomly incorporated into the rice genome and not near the $L R K$ gene cluster, we concluded that $L A I R$ is a crucial trans-acting regulator of $L R K$ transcription. We also detected an intriguing gene expression pattern in LAIR-overexpressing rice lines. Specifically, there were differences in the expression level changes among the $L R K$ genes (Fig. 3a). These differences were also observed in the luciferase reporter assays (Fig. 3c). Therefore, LAIR regulates $L R K s$ expression with diverse effects among genes.

The mechanisms underlying the lncRNA-associated regulation of gene expression are very diverse. Recent reports described a crucial role for lncRNAs involving epigenetic modifications to specific genomic loci, which then regulate the expression of neighbouring protein-coding genes ${ }^{6,30,44}$. Given that LAIR activates the expression of $L R K$ genes, we analyzed the histone 
a
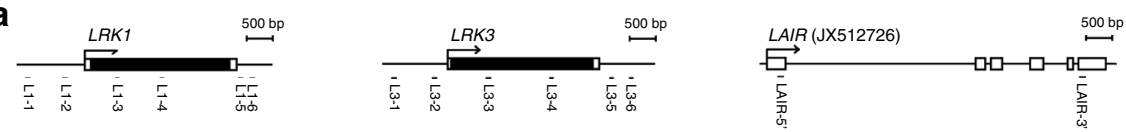

b $\square$ WT $\square$ LAIR-1 $\square$ LAIR-2
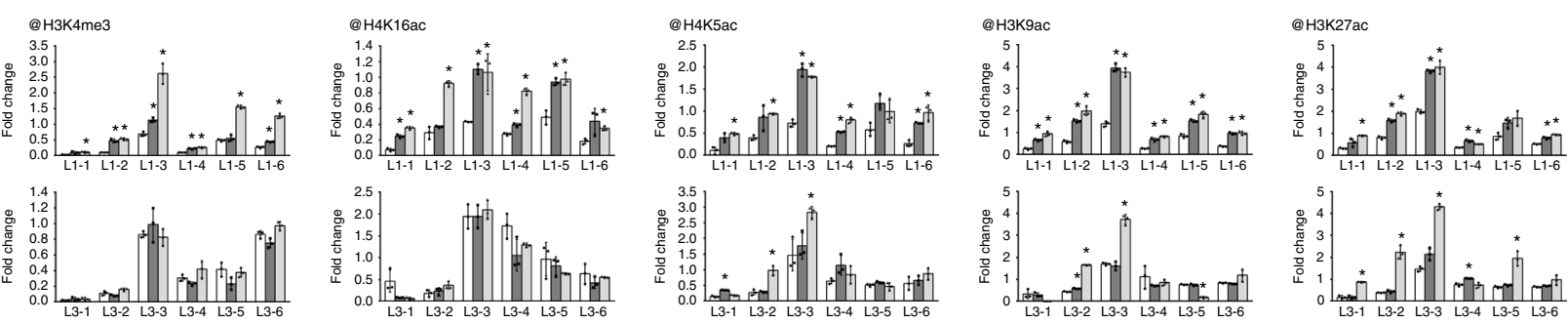

C $\square$ WT $\square$ Anti-LAIR-1 $\square$ Anti-LAIR-2
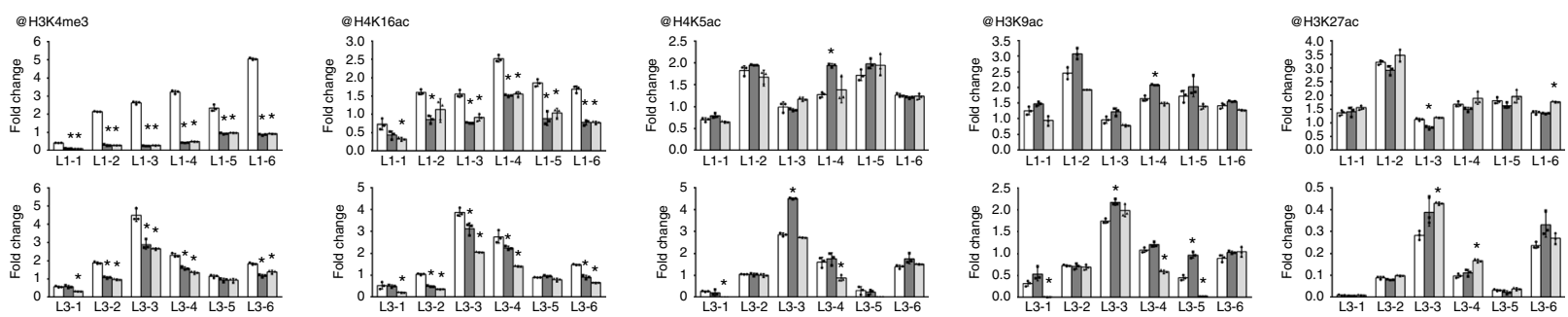

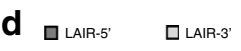

e $\square$ wT

$\square$ OsWDR5-1
口 OsWDR5-2

C OsMOF-1
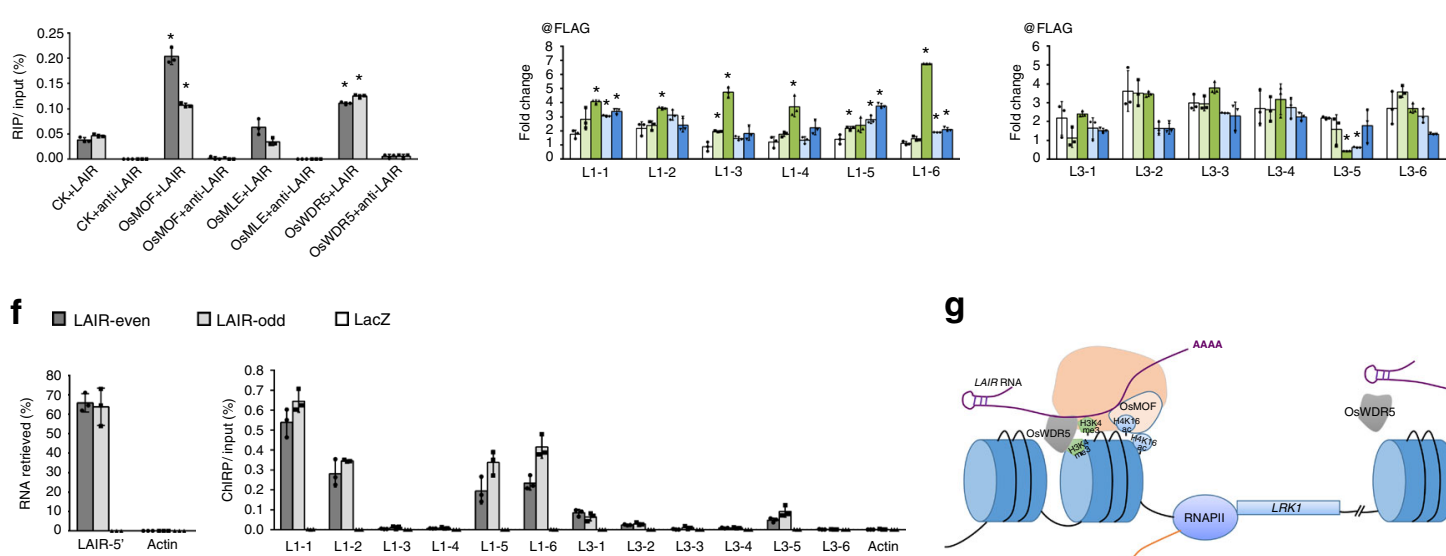

口LAIR-odd $\square$ LacZ

\section{g}
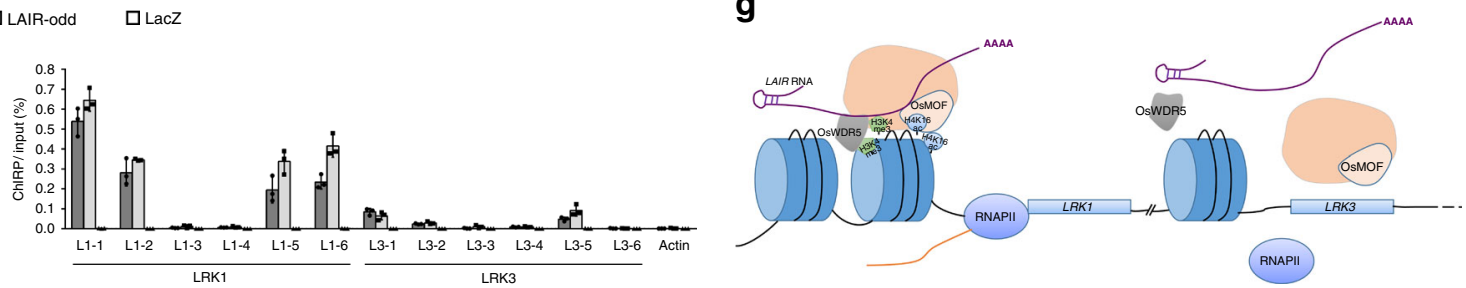

Fig. 4 Molecular processes involving LAIR RNA, epigenetic modification proteins and chromatin of $L R K$ genes. a Schematic representation of $L R K 1$, $L R K 3$, and LAIR (JX512726) gene structure and primers position indicating the regions examined in the ChIP, RIP, and ChIRP assays of b-f. Black boxes indicate open reading frame. $\mathbf{b}, \mathbf{c}$ ChIP assays of $L R K$ loci. The ChIP analyses were completed for the $L R K 1$ and $L R K 3$ chromatin regions using antibodies against H3K4me3, H4K5ac, H3K9ac, H4K16ac, and H3K27ac in rice wild-type (WT) and LAIR-overexpressing (LAIR-1 and LAIR-2) lines (b) or antisense RNAiLAIR lines (Anti-LAIR-1 and Anti-LAIR-2) (c). OsActin was used as a reference control and for data normalization. d RIP assays of epigenetic modificationassociated proteins (OsMOF, OsMLE, and OsWDR5) confirmed the interaction with LAIR. The proteins were fused to the FLAG tag. CK: empty vector control with only the FLAG tag. The RIP was completed in rice protoplasts that were co-infiltrated with fusion proteins vector or the control, and the vector with the 35S::LAIR or 35S::anti-LAIR. e ChIP assays of modification-associated proteins on LRK loci. The analyses were completed for LRK1 and LRK3 using an anti-FLAG antibody in rice wild-type (WT) as well as OsWDR5-FLAG (OsWDR5-1 and OsWDR5-2) and OsMOF-FLAG (OsMOF-1 and OsMOF-2) transgenic lines. OsActin was used as a reference control and for data normalization. $\mathbf{f}$ ChIRP assays of LAIR on LRK loci. ChIRP enriched $\sim 65 \%$ of LAIR RNA in both odd and even probes pool, with undetectable OsActin (Actin) (left panel). Immunoprecipitated DNA genomic region analyzed by ChIRP-qPCR showed the remarkable interaction of LAIR with the $5^{\prime}$ and $3^{\prime}$ untranslated regions of $L R K 1$ compare with $L R K 3$ (right panel). Probes that targeted LacZ mRNA were served as negative controls. b-f Student's $t$-test: ${ }^{\star} P<0.05$. Data are presented as the mean \pm standard deviation $(n=3)$. $\mathbf{g} A$ proposed model of LAIR during the regulation of LRKs. LAIR interacts with epigenetic regulation proteins OsMOF and OsWDR5; both IncRNA and epigenetic modification proteins locate to $L R K 1$ genomic region; and LAIR overexpression results in changes in histone marks (H3K4me3 and H4K16ac) and activating transcription of $L R K 1$. The process is not executed on $L R K 3$ genomic region. This difference may contribute to the observed differential regulation of $L R K$ genes 
modification status of $L R K$ loci. We observed that $\mathrm{H} 3 \mathrm{~K} 4 \mathrm{me} 3$ and $\mathrm{H} 4 \mathrm{~K} 16 \mathrm{ac}$ were enriched at $L R K 1$ in LAIR overexpression lines, implying that epigenetic changes to specific $L R K$ loci were associated with altered LAIR expression (Fig. 4b). Hundreds of lncRNAs have been confirmed to interact with multiple protein partners, suggesting lncRNAs influence gene expression by targeting chromatin remodelers to specific genomic regions as part of a molecular scaffold ${ }^{6,11}$. RNA-binding proteins OsMOF and OsWDR5, which have shown to associate with the H3K4me3 and H4K16ac histone modification complex, were detected significantly binding with LAIR (Fig. 4d and Supplementary Fig. 4). Additionally, OsMOF and OsWDR5 were enriched at the LRK1 locus (Fig. 4e). Finally, we found evidence on LAIR RNA binding with LRK1 genomic locus (Fig. 4f). Thus, LAIR could interact with epigenetic regulation proteins OsMOF and OsWDR5; both lncRNA and epigenetic modification proteins could locate to specific $L R K$ genes; and LAIR overexpression resulted in altered $L R K$ genes expression and changes in histone marks (Fig. $4 \mathrm{~g}$ ). However, current evidence do not support that LAIR acts through LRKs to regulate grain yield and it is not clear whether the changes in histone marks are the cause of LRKs expression or occurring as a consequence.

While the lncRNA activities continue to be clarified, the mechanisms regulating plant lncRNAs remain relatively uncharacterized. As a key histone acetyltransferase in mammalian cells, MOF has been well studied in human and fruit fly, but the research of MOF in rice is almost zero. There are currently no published reports describing lncRNA-regulated gene expression associated with the histone modification protein OsMOF in rice. The NSL complex is a universal and sex-independent major regulator of housekeeping genes ${ }^{37,45,46}$. Because two of its crucial components (OsMOF and OsWDR5) interact with LAIR, it is likely that $L A I R$ functions are associated with the evolutionarily conserved NSL complex. However, additional research is required to elucidate this potential relationship.

The biological features of IncRNAs have attracted widespread interest, and important advances have been made in nearly all cellular systems or species that have been studied at the genome level. Regarding applied research, current efforts are mostly related to the potential utility of lncRNAs in cancer therapeutics, regenerative medicine involving stem cell technology, and biomarker development for molecular interventions ${ }^{42,47-50}$. However, studies on the application of lncRNAs in plants, especially for crop breeding, remain limited. To satisfy future food and bio-fuel demands, innovative molecular methods to improve crop yields must be developed. The rice LAIR described herein may be useful for enhancing plant growth and grain yield. Relative to the wild-type plants, the LAIR-overexpressing transgenic rice lines produced larger primary panicles and had more panicles per plant, which likely contributed to the considerable increase in total grain yield (Fig. 1b-e). As a new genetic element identified at a QTL, LAIR may represent a new option for introducing stable and heritable increases of grain yield in rice. Rice is considered a model cereal crop because of its well-established genetic background and molecular breeding system. Thus, it may be relevant for characterizing lncRNAs in terms of the associated molecular mechanisms and individual gene expression levels, as well as for the application of lncRNAs in crop breeding. Future studies should aim to characterize the molecular mechanism underlying $L A I R$-regulated gene expression with diverse effects, and explore the potential application of LAIR for improving crop yield.

\section{Methods}

Plant materials and gene transformation. O. sativa ssp. indica rice cultivar MingHui63 (MH63) was used in this study. Additionally, the binary vector pCAMBIA1304 (Center for the Application of Molecular Biology to International
Agriculture), which carries the kanamycin- and hygromycin-resistance genes for screening transformed bacteria and plants, respectively, was assembled. The LAIR isoform of JX512726 was cloned and used to construct 35S:LAIR transgenic line. To induce rice embryogenic calli, dehusked MH63 rice seeds were surface-sterilized by soaking in $70 \%(\mathrm{v} / \mathrm{v})$ ethanol for $5-10 \mathrm{~min}$ and then $0.1 \%(\mathrm{w} / \mathrm{v}) \mathrm{HgCl}_{2}$ for 20-30 min. Seeds were then washed 5-7 times with sterile distilled water and sown on agar-solidified Murashige and Skoog medium. About 20 days later, the calli were bombarded using a gene gun and selected after a 2-week exposure to $50 \mathrm{mg} \mathrm{l}^{-1}$ hygromycin. The hygromycin-resistant calli were regenerated and grown ( $\mathrm{T}_{0}$ lines). A total of 10-20 independent $\mathrm{T}_{0}$ lines were tested to confirm they were successfully transformed. The hygromycin gene was amplified by a polymerase chain reaction (PCR) with genomic DNA used as the template.

All rice materials were grown in a greenhouse at $28^{\circ} \mathrm{C}$ under a $14 \mathrm{~h}$-light $/ 10 \mathrm{~h}$ dark photoperiod or in test fields in Shanghai $\left(31^{\circ} 11^{\prime} \mathrm{N}, 121^{\circ} 29^{\prime} \mathrm{E}\right)$ and Sanya $\left(18^{\circ}\right.$ $\left.14^{\prime} \mathrm{N}, 109^{\circ} 31^{\prime} \mathrm{E}\right)$, China.

RNA isolation and quantitative real-time PCR. Rice lines were grown in a greenhouse until reaching the three-leaf stage, after which the RNAprep Pure Plant Kit (Tiangen, Beijing, China) was used to extract total RNA. First-strand cDNA was synthesized using the PrimeScript ${ }^{\text {tw }}$ RT Reagent Kit (Takara, Otsu, Japan). A quantitative real-time (qRT)-PCR was conducted using SYBR ${ }^{\circledR}$ Premix Ex Taq ${ }^{\mathrm{m}}$ (Takara) and the CFX96 Real-Time PCR Detection system (Bio-Rad, Hercules, CA, USA). The qRT-PCR program was as follows: 40 cycles of $95^{\circ} \mathrm{C}$ for $5 \mathrm{~s}$ and $60^{\circ} \mathrm{C}$ for $30 \mathrm{~s}$. Melting and standard curves were prepared and analyzed. OsActin was used as a reference gene for normalizing transcript levels. Details regarding the primers used in this study are provided in Supplementary Data 1.

Rapid amplification of cDNA ends. Total RNA was extracted from wild-type MH63 plants at the three-leaf stage as described above. The 3' RACE analysis was completed using the $3^{\prime}$-Full RACE Core Set with PrimeScript ${ }^{\mathrm{m}}$ RTase (Takara), while the $5^{\prime}$ RACE analysis was completed with the $5^{\prime}$-Full RACE Kit (Takara). The $5^{\prime}$ and $3^{\prime}$ cDNA fragments were amplified using gene-specific primers (Supplementary Data 1). Additionally, the RACE products were subsequently characterized by PCR or sequencing.

Rice protoplasts generation and gene expression analysis. A 1.5-kb sequence upstream of the ATG start codon $(-1500$ to -1$)$, which corresponded to the $L R K$ promoter region, was amplified with the LRKn promoter primers (Supplementary Data 1). The amplified fragment was cloned into the pGreenII 0800-LUC ${ }^{51}$ dual luciferase (LUC) assay system transient expression vector so that it was followed by the $L U C$ reporter gene.

Leaves from rice plants at the three-leaf stage were treated with cellulase and macerozyme to generate protoplasts, which were transiently transformed with the prepared vectors using a $40 \%$ polyethylene glycol solution ${ }^{52}$. After a $12-16 \mathrm{~h}$ incubation at $28^{\circ} \mathrm{C}$ in darkness, the protoplast lysate was collected and tested using the Dual-Luciferase ${ }^{\varpi}$ Reporter Assay System (Promega, Madison, WI, USA) and the Synergy ${ }^{\text {rm }} 2$ Multi-Mode Reader (BioTek, Winooski, VT, USA). The expression levels of the $L U C$ reporter gene under the control of the target promoter and the Renilla luciferase $(R E N)$ reporter gene under the control of the CaMV 35S promoter were analyzed. The LUC/REN signal ratio can be used to determine the relative ability of ncRNAs to induce the expression of genes under the control of different promoter sequences.

Chromatin immunoprecipitation assay. Two-week-old rice seedlings were harvested and immersed in a fixing buffer [0.4 M sucrose, $10 \mathrm{mM}$ Tris- $\mathrm{HCl}, \mathrm{pH} 8.0,1$ mM EDTA, pH 8.0, 1\% formaldehyde, and a protease inhibitor cocktail tablet (Roche, Indianapolis, IN, USA)] under vacuum conditions for 15-20 min. The seedlings were incubated for an additional $5 \mathrm{~min}$ after $0.1 \mathrm{M}$ glycine was added. Seedlings were rinsed 5 times with distilled water and then frozen with liquid nitrogen. Fixed seedlings were ground to a powder and resuspended in $30 \mathrm{ml}$ lysis buffer (50 mM HEPES, pH 7.5, 1 mM EDTA, pH 8.0, $150 \mathrm{mM} \mathrm{NaCl}, 1 \%$ Triton X100, $10 \%$ glycerol, $5 \mathrm{mM} \beta$-mercaptoethanol, and a protease inhibitor cocktail tablet). Solutions were filtered through a $100-\mu \mathrm{m}$ nylon mesh and centrifuged at $1500 \times g$ for $20 \mathrm{~min}$. The pelleted chromatin was resuspended and sheared by sonication to produce approximately 200-bp fragments. After a centrifugation at $13,000 \times g$ for $10 \mathrm{~min}$, the supernatants were transferred to new tubes and the appropriate antibodies were added $[2 \mu \mathrm{l}(1: 600)$ anti-trimethyl-H3K4 (Cat. No. ab8580), anti-acetyl-H4K5 (Cat. No. ab51997), anti-acetyl-H3K9 (Cat. No. ab10812), anti-acetyl-H4K16 (Cat. No. ab109463), or anti-acetyl-H3K27 (Cat. No. ab4729); Abcam, Cambridge, UK or $3 \mu \mathrm{l}$ (1:400) monoclonal anti-FLAG M2 (Cat. No. F1804); Sigma-Aldrich, St. Louis, MO, USA]. Samples were rotated overnight, mixed with $40 \mu \mathrm{l}$ Magnetic Protein A Beads, and incubated for another 1-1.5 h. Beads were washed with low salt, high salt, $\mathrm{LiCl}$, and TE buffers $(1 \mathrm{ml}$ each). Immunocomplexes were eluted from the beads with $500 \mu$ l elution buffer ( $1 \%$ SDS and $0.1 \mathrm{M} \mathrm{NaHCO}_{3}$, with $20 \mu \mathrm{l} 5 \mathrm{M} \mathrm{NaCl}$ in each tube). Additionally, crosslinks were reversed by incubating samples at $65^{\circ} \mathrm{C}$ for at least $5-6 \mathrm{~h}$. Residual proteins were degraded by the addition of a solution consisting of $10 \mathrm{mM}$ EDTA, pH 8.0, 40 $\mathrm{mM}$ Tris- $\mathrm{HCl}, \mathrm{pH} 8.0$, and $2 \mu \mathrm{l}$ Proteinase $\mathrm{K}$ followed by an incubation at $45^{\circ} \mathrm{C}$ for $1 \mathrm{~h}$. Moreover, DNA was purified and resuspended in $100 \mu \mathrm{l}$ sterile distilled water. 
A qRT-PCR was conducted to assess the enrichment of immunoprecipitated DNA during the ChIP experiments. Details regarding the gene-specific primers are listed in Supplementary Data 1. OsActin was used as an internal standard for normalizing data.

RNA immunoprecipitation assay. The full-length coding sequences for genes associated with epigenetic modifications (OsMOF, OsMLE, and OsWDR5) were cloned from rice MH63 cDNA and fused in-frame with the gene encoding the FLAG tag under the control of the 35S CaMV promoter in the pCAMBIA1306 vector. Rice protoplasts were co-infiltrated with this vector or the control (i.e., empty vector encoding the FLAG tag) and the vector with the 35S::LAIR or $35 \mathrm{~S}::$ anti-LAIR vector. After a $12-16$-h incubation at $28^{\circ} \mathrm{C}$ in darkness, protoplasts were harvested for RIP assays.

The RIP assays were conducted using the EZ-Magna RIP ${ }^{\mathrm{ms}}$ Kit (Millipore, Bedford, MA, USA). Monoclonal anti-FLAG M2 (Cat. No. F1804, Sigma-Aldrich, St. Louis, MO, USA) was added ( $5 \mu \mathrm{l})$ to each reaction. Purified RNA was used as the template to prepare first-strand cDNA with the PrimeScript ${ }^{\text {tw }}$ RT Reagent Kit. A qRT-PCR was completed using gene-specific primers (Supplementary Data 1) to assess the enrichment of the immunoprecipitated RNA, with OsActin serving as the reference control. The RIP efficiency of the protoplasts transformed with the control vector was used to normalize data.

Chromatin isolation by RNA purification. Rice protoplasts were generated from MH63 and harvested for ChIRP assays. The ChIRP assays were conducted using the EZ-Magna ChIRP ${ }^{\mathrm{m}}$ RNA Interactome Kit (Millipore, Bedford, MA, USA). Sonication was performed in ice-cold water bath at setting with high-power, $30 \mathrm{~s}$ ON, $30 \mathrm{~s}$ OFF pulse intervals for $2 \mathrm{~h}$ (total process time). Anti-sense biotinylated DNA tiling probes for selective retrieval of RNA target were designed by online probe design program (www.singlemoleculefish.com), and details are provided in Supplementary Table 1. For LAIR, probes were separated into two groups (even and odd), which served as internal control for each other. Probes that targeted LacZ mRNA, which absent from rice cell, were used as negative control probes ${ }^{14}$.

After ChIRP and RNA/DNA isolation, qRT-PCR was completed using genespecific primers (Supplementary Data 1 ) to assess the enrichment of the retrieved RNA and immunoprecipitated DNA, with OsActin serving as a negative control gene.

\section{Data availability}

Sequence data in this study have been deposited in the GenBank database and are available with the following accession codes: JX512717, JX512718, JX512719, JX512720, JX512721, JX512722, JX512723, JX512724, JX512725, JX512726, JX512727, and JX512728. The data that support the findings of this study are available from the corresponding authors upon reasonable request.

Received: 4 December 2017 Accepted: 26 July 2018

Published online: 29 August 2018

\section{References}

1. Khush, G. S. Origin, dispersal, cultivation and variation of rice. Plant Mol. Biol. 35, 25-34 (1997).

2. Gale, M. D. \& Devos, K. M. Comparative genetics in the grasses. Proc. Natl Acad. Sci. USA 95, 1971-1974 (1998).

3. Sasaki, T. \& Burr, B. International Rice Genome Sequencing Project: the effort to completely sequence the rice genome. Curr. Opin. Plant Biol. 3, 138-141 (2000).

4. Djebali, S. et al. Landscape of transcription in human cells. Nature $\mathbf{4 8 9}$ 101-108 (2012)

5. Derrien, T. et al. The GENCODE v7 catalog of human long noncoding RNAs: analysis of their gene structure, evolution, and expression. Genome Res. 22, 1775-1789 (2012).

6. Rinn, J. L. \& Chang, H. Y. Genome regulation by long noncoding RNAs. Annu. Rev. Biochem. 81, 145-166 (2012).

7. Wang, H. et al. Genome-wide identification of long noncoding natural antisense transcripts and their responses to light in Arabidopsis. Genome Res. 24, 444-453 (2014).

8. Geisler, S. \& Coller, J. RNA in unexpected places: long non-coding RNA functions in diverse cellular contexts. Nat. Rev. Mol. Cell Biol. 14, 699-712 (2013).

9. Wang, X., Song, X., Glass, C. \& Rosenfeld, M. G. The long arm of long noncoding RNAs: roles as sensors regulating gene transcriptional programs. Cold Spring Harb. Perspect. Biol. 3, a003756 (2011).

10. Guil, S. \& Esteller, M. Cis-acting noncoding RNAs: friends and foes. Nat. Struct. Mol. Biol. 19, 1068-1075 (2012).

11. Khalil, A. M. et al. Many human large intergenic noncoding RNAs associate with chromatin-modifying complexes and affect gene expression. Proc. Natl Acad. Sci. USA 106, 11667-11672 (2009).
12. Bernstein, E. \& Allis, C. D. RNA meets chromatin. Genes Dev. 19, 1635-1655 (2005).

13. Rinn, J. L. et al. Functional demarcation of active and silent chromatin domains in human HOX loci by non-coding RNAs. Cell 129, 1311-1323 (2007).

14. Chu, C., Qu, K., Zhong, F., Artandi, S. \& Chang, H. Genomic maps of long noncoding RNA occupancy reveal principles of RNA-chromatin interactions. Mol. Cell 44, 667-678 (2011).

15. Lakhotia, S. C. Divergent actions of long noncoding RNAs on X-chromosome remodelling in mammals and Drosophila achieve the same end result: dosage compensation. J. Genet. 94, 575-584 (2015).

16. Meller, V. H., Wu, K. H., Roman, G., Kuroda, M. I. \& Davis, R. L. roX1 RNA paints the $\mathrm{X}$ chromosome of male Drosophila and is regulated by the dosage compensation system. Cell 88, 445-457 (1997).

17. Park, S.-W. et al. An evolutionarily conserved domain of roX2 RNA is sufficient for induction of H4-Lys16 acetylation on the Drosophila X chromosome. Genetics 177, 1429-1437 (2007)

18. Quinn, J. J. et al. Rapid evolutionary turnover underlies conserved lncRNA-genome interactions. Genes Dev. 30, 191-207 (2016).

19. Berry, S. \& Dean, C. Environmental perception and epigenetic memory: mechanistic insight through FLC. Plant J 83, 133-148 (2015).

20. Li, S., Yamada, M., Han, X., Ohler, U. \& Benfey, P. N. High-resolution expression map of the Arabidopsis root reveals alternative splicing and lincRNA regulation. Dev. Cell 39, 508-522 (2016).

21. Matzke, M. A. \& Mosher, R. A. RNA-directed DNA methylation: an epigenetic pathway of increasing complexity. Nat. Rev. Genet. 15, 394-408 (2014).

22. Wang, D. et al. Transposable elements (TEs) contribute to stress-related long intergenic noncoding RNAs in plants. Plant J 90, 133-146 (2017).

23. Wang, Y. et al. Arabidopsis noncoding RNA mediates control of photomorphogenesis by red light. Proc. Natl Acad. Sci. USA 111, 10359-10364 (2014).

24. Zhang, Y. C. et al. Genome-wide screening and functional analysis identify a large number of long noncoding RNAs involved in the sexual reproduction of rice. Genome Biol. 15, 512 (2014).

25. He, G. et al. Haplotype variation in structure and expression of a gene cluster associated with a quantitative trait locus for improved yield in rice. Genome Res. 16, 618-626 (2006)

26. Bateman, A. et al. The Pfam protein families database. Nucleic Acids Res. 32, D138-D141 (2004).

27. Letunic, I. et al. SMART 5: domains in the context of genomes and networks. Nucleic Acids Res. 34, D257-D260 (2006).

28. Kong, L. et al. CPC: assess the protein-coding potential of transcripts using sequence features and support vector machine. Nucleic Acids Res. 35, W345-W349 (2007).

29. Zha, X. et al. Over-expression of the rice LRK1 gene improves quantitative yield components. Plant Biotechnol. J 7, 611-620 (2009).

30. Mercer, T. R., Dinger, M. E. \& Mattick, J. S. Long non-coding RNAs: insights into functions. Nat. Rev. Genet. 10, 155-159 (2009).

31. $\mathrm{Du}, \mathrm{Z}$. et al. Genome-wide analysis of histone modifications: H3K4me2, $\mathrm{H} 3 \mathrm{~K} 4 \mathrm{me} 3, \mathrm{H} 3 \mathrm{~K} 9 \mathrm{ac}$, and H3K27ac in Oryza sativa L. Japonica. Mol. Plant 6, 1463-1472 (2013)

32. Komiya, R., Ikegami, A., Tamaki, S., Yokoi, S. \& Shimamoto, K. Hd3a and RFT1 are essential for flowering in rice. Development 135, 767-774 (2008).

33. Wang, K. C. et al. A long noncoding RNA maintains active chromatin to coordinate homeotic gene expression. Nature 472, 120-124 (2011).

34. Xiao, J. et al. Requirement of histone acetyltransferases HAM1 and HAM2 for epigenetic modification of FLC in regulating flowering in Arabidopsis. J. Plant Physiol. 170, 444-451 (2013).

35. Taipale, M. et al. hMOF histone acetyltransferase is required for histone $\mathrm{H} 4$ lysine 16 acetylation in mammalian cells. Mol. Cell. Biol. 25, 6798-6810 (2005).

36. Conrad, T. \& Akhtar, A. Dosage compensation in Drosophila melanogaster: epigenetic fine-tuning of chromosome-wide transcription. Nat. Rev. Genet. 13, 123-134 (2012).

37. Raja, S. J. et al. The nonspecific lethal complex is a transcriptional regulator in Drosophila. Mol. Cell 38, 827-841 (2010).

38. Dias, J. et al. Structural analysis of the KANSL1/WDR5/KANSL2 complex reveals that WDR5 is required for efficient assembly and chromatin targeting of the NSL complex. Genes Dev. 28, 929-942 (2014).

39. Gilfillan, G. D., Dahlsveen, I. K. \& Becker, P. B. Lifting a chromosome: dosage compensation in Drosophila melanogaster. FEBS Lett. 567, 8-14 (2004).

40. Lucchesi, J. C. Dosage compensation: roX marks the spot. Curr. Biol. 9 , R807-R808 (1999).

41. Yang, Y. W. et al. Essential role of IncRNA binding for WDR5 maintenance of active chromatin and embryonic stem cell pluripotency. eLife 3, e02046 (2014). 
42. Rao, M. R. S. Long Non Coding RNA Biology. (Springer Nature, Singapore, 2017).

43. Luo, S. et al. Divergent lncRNAs regulate gene expression and lineage differentiation in pluripotent cells. Cell Stem Cell 18, 637-652 (2016).

44. Mondal, T., Rasmussen, M., Pandey, G. K., Isaksson, A. \& Kanduri, C. Characterization of the RNA content of chromatin. Genome Res. 20, 899-907 (2010).

45. Mendjan, S. et al. Nuclear pore components are involved in the transcriptional regulation of dosage compensation in Drosophila. Mol. Cell 21, 811-823 (2006).

46. Lam, K. C. et al. The NSL complex regulates housekeeping genes in Drosophila. PLoS Genet. 8, e1002736 (2012).

47. Takahashi, K., Yan, I. K., Wood, J., Haga, H. \& Patel, T. Involvement of extracellular vesicle long non-coding RNA (linc-VLDLR) in tumor cell responses to chemotherapy. Mol. Cancer Res. 12, 1377-1387 (2014).

48. Han, L. C. \& Chen, Y. Small and long non-coding RNAs: novel targets in perspective cancer therapy. Curr. Genomics 16, 319-326 (2015).

49. Meng, L. et al. Towards a therapy for Angelman syndrome by reduction of a long non-coding RNA. Nature 518, 409-412 (2015).

50. Parasramka, M. A., Maji, S., Matsuda, A., Yan, I. K. \& Patel, T. Long noncoding RNAs as novel targets for therapy in hepatocellular carcinoma. Pharmacol. Ther. 161, 67-78 (2016).

51. Hellens, R. P. et al. Transient expression vectors for functional genomics, quantification of promoter activity and RNA silencing in plants. Plant Methods 1, 13 (2005).

52. Yoo, S. D., Cho, Y. H. \& Sheen, J. Arabidopsis mesophyll protoplasts: a versatile cell system for transient gene expression analysis. Nat. Protoc. 2 1565-1572 (2007)

\section{Acknowledgements}

We thank Prof. Aiwu Dong for her valuable discussions and advices, Dr. Bing Liu for his technical assistance with ChIP assay. This work was supported by grants from the National Key Research and Development Program of China (2016YFD0100902), the National Natural Science Foundation of China (31671263), China Postdoctoral Science Foundation Funded Project (KLH1322099), and the Basic Application Research Program from the Shanghai Municipal Agriculture Commission (2014-7-1-2).

\section{Author contributions}

Y.W. designed the study, carried out the experiments, analyzed data, and wrote the paper. X.L. and X.Z. helped with rice plants management in test fields. F.S. performed protoplasts experiments. J.H. helped with the experimental design. W.S. and J.Y. designed the study, analyzed data, and wrote the paper.

\section{Additional information}

Supplementary Information accompanies this paper at https://doi.org/10.1038/s41467018-05829-7.

Competing interests: The authors declare no competing interests.

Reprints and permission information is available online at http://npg.nature.com/ reprintsandpermissions/

Publisher's note: Springer Nature remains neutral with regard to jurisdictional claims in published maps and institutional affiliations.

(c) (i) Open Access This article is licensed under a Creative Commons Attribution 4.0 International License, which permits use, sharing, adaptation, distribution and reproduction in any medium or format, as long as you give appropriate credit to the original author(s) and the source, provide a link to the Creative Commons license, and indicate if changes were made. The images or other third party material in this article are included in the article's Creative Commons license, unless indicated otherwise in a credit line to the material. If material is not included in the article's Creative Commons license and your intended use is not permitted by statutory regulation or exceeds the permitted use, you will need to obtain permission directly from the copyright holder. To view a copy of this license, visit http://creativecommons.org/ licenses/by/4.0/.

(C) The Author(s) 2018 